\title{
Predator-prey dynamics with intraspecific competition and an Allee effect in the predator population
}

\author{
Erin N. Bodine ${ }^{a}$ and Anne E. Yust ${ }^{b}$ \\ ${ }^{a}$ Department of Mathematics \& Computer Science, Rhodes College, Memphis, TN, USA; ${ }^{b}$ Department of \\ Natural Sciences \& Mathematics, The New School, Eugene Lang College of Liberal Arts, New York, NY, USA
}

\begin{abstract}
The study of the Allee effect on the stability of equilibria of predatorprey systems is of recent interest to mathematicians, ecologists, and conservationists. Many theoretical models that include the Allee effect result in an unstable coexistence equilibrium. However, empirical evidence suggests that predator-prey systems exhibiting densitydependent growth at small population densities still can achieve coexistence in the long term. We review an often cited model that incorporates an Allee effect in the predator population resulting in an unstable coexistence equilibrium, and then present a novel extension to this model which includes a term modeling intraspecific competition within the predator population. The additional term penalizes predator population growth for large predator to prey density ratios. We use equilibrium analysis to define the regions in the parameter space where the coexistence equilibrium is stable, and show that there exist biologically reasonable parameter sets which produce a stable coexistence equilibrium for our model.
\end{abstract}

\section{ARTICLE HISTORY}

Received 10 August 2016

Accepted 7 January 2017

\section{KEYWORDS}

Predator-prey dynamics; Allee effect; intraspecific competition; differential equation model; stability analysis; numerical simulation

\section{Introduction}

In the 1920s, Lotka (1925) and Volterra (1926) independently proposed what are now referred to as the Lotka-Volterra equations,

$$
\begin{aligned}
& \frac{\mathrm{d} N}{\mathrm{~d} t}=r_{1} N-\delta N P, \\
& \frac{\mathrm{d} P}{\mathrm{~d} t}=-r_{2} P+\theta N P,
\end{aligned}
$$

where $N(t)$ and $P(t)$ are the populations sizes or densities of the prey and predator populations, respectively. The prey population exhibits exponential growth (with intrinsic growth rate of $r_{1}>0$ ) in the absence of the predator, but the growth rate of the prey population is reduced by interaction with predators as governed by the functional response term $\delta N P$. The predator population exhibits exponential decay (with intrinsic decay rate

CONTACT Erin N. Bodine bodinee@rhodes.edu

(C) 2017 The Author(s). Published by Informa UK Limited, trading as Taylor \& Francis Group

This is an Open Access article distributed under the terms of the Creative Commons Attribution License (http://creativecommons.org/ licenses/by/4.0/), which permits unrestricted use, distribution, and reproduction in any medium, provided the original work is properly cited. 
of $r_{2}>0$ ) in the absence of a prey source, and the growth rate of the predator population is increased by interaction with prey as governed by the numerical response term $\theta N P$. The interaction parameters $\delta$ and $\theta$ are both assumed to be positive constants.

The Lotka-Volterra system can be described more generally as

$$
\begin{aligned}
\frac{\mathrm{d} N}{\mathrm{~d} t} & =g(N) N-f_{1}(N, P), \\
\frac{\mathrm{d} P}{\mathrm{~d} t} & =m(P) P+f_{2}(N, P),
\end{aligned}
$$

where $g(N)$ is the per capita growth rate of the prey population in the absence of the predator population, $m(P)$ is the per capita growth rate of the predator population (or mortality rate if $m(P)<0)$ in the absence of the prey population and $f_{1}(N, P)$ and $f_{2}(N, P)$ are the functional response and numerical response terms, respectively, to interaction with the other population. For System $(1), g(N)=r_{1}, m(P)=-r_{2}, f_{1}(N, P)=\delta N P$, and $f_{2}(N, P)=\theta N P$. Note, when $m(P)<0$, any growth in the predator population is a result of its numerical response to its prey.

Many adaptations of the Lotka-Volterra model have been studied since it was originally proposed in the 1920s. One adaptation has been to include an Allee effect in either the predator or prey population (Aulisa \& Jang, 2014; Flores \& González-Olivares, 2014; Zhou, Liu, \& Wang, 2005 for some examples). The Allee effect is defined as a positive relationship between population density and fitness (Allee, 1941; Boukal \& Berec, 2009; Courchamp, Berec, \& Gascoigne, 2009). The Allee effect is thought to be present in many populations and can be caused by a variety of factors. Populations with small densities can experience magnified threats to their fitness and survival, which can cause a reduction in a population's per capita growth rate. The effect is described as either being weak or strong, but only the strong Allee effect exhibits a negative per capita growth rate for sufficiently low population densities.

There are many ways that an Allee effect can be present in a predator-prey system. For example, predators can drive an Allee effect in prey by affecting individual probability of survival (Gascoigne \& Lipcius, 2004). Alternatively, predator density can affect the per capita benefit of prey to the predator population (Zhou et al., 2005). In the first instance, the Allee effect is acting upon the prey population by reducing the prey per capita growth rate, $g(N)$. In the latter case, the Allee effect is acting upon the predator population by reducing the numerical response of the predator population to its prey source, $f_{2}(N, P)$. This effect could occur in systems where multiple predators are required in order to make a kill. For instance, African wild dogs experience a decrease in per capita net energy gain when pack size is reduced. Causes of this decrease could include the increase in energy expended per individual during a hunt, or a shorter post-hunt feeding period if there are not enough individuals to guard their feeding packmates (Gusset \& Macdonald, 2010).

In Section 2, we describe a predator-prey model proposed by Zhou et al. (2005) which incorporates an Allee effect into the predator population through the numerical response of the predator to its prey, $f_{2}(N, P)$. However, for all biologically reasonable parameter sets, this model produces unstable oscillations leading to boom and bust cycles with increasing amplitudes which eventually become biologically untenable. In response, in Section 3, we propose a modified version of the Zhou et al. model in which intraspecific 
competition between predators for prey curtails the growth of the predator population and allows for biologically tenable oscillations and the presence of a stable coexistence equilibrium. Intraspecific competition between predators for prey occurs when the ratio of predators to prey is sufficiently large, causing individuals in the predator population to experience reduced fitness due to lack of sustenance (Purves, Sadava, Orians, \& Heller, 2001). This intensified competition has been shown in blue crab populations where they express agonistic behaviour, which results in injury when available prey is scarce (Clark, Wolcott, Wolcott, \& Hines, 1999). More extreme intraspecific competition has manifested in intraspecific predation in a variety of predator populations due to scarcity of another food source (Fox, 1975; Polis, 1981). The result of the intraspecific competition between predators is that the numerical response of the predator population to its prey becomes negative, $f_{2}(N, P)<0$. In Section 3, we provide a detailed description of the model and its equilibrium points, and then we derive the stability conditions for the coexistence equilibrium. In Section 4, we compare our proposed model's coexistence equilibrium and its stability with that of the Zhou et al. model and the Lotka-Volterra model, concluding with a discussion of some of the limitations and possible extensions of our model.

\section{Predator-prey model with a predator population Allee effect}

An Allee effect can be incorporated into the predator population of System (2) by modifying the numerical response of the predators to interaction with the prey, $f_{2}(N, P)$, such that $f_{2}$ is decreased for small $P$. Zhou et al. (2005) examines the following model

$$
\begin{aligned}
& \frac{\mathrm{d} N}{\mathrm{~d} t}=r_{1} N-\delta N P, \\
& \frac{\mathrm{d} P}{\mathrm{~d} t}=-r_{2} P+\theta N P\left(\frac{P}{P+B}\right),
\end{aligned}
$$

where $r_{1}, r_{2}, \delta$ and $\theta$ are positive valued and as described for the Lotka-Volterra model (1), and the parameter $B>0$ is defined as the Allee effect constant. Note, the larger the value of $B$, the lower the per captia growth rate of the predator population. Model (3) is the same as the Lotka-Volterra system in Model (1), but the predator population's numerical response term is decreased as compared to the Lotka-Volterra system since $\frac{P}{P+B}<1$. Furthermore, while any ratio of $B / P$ will reduce the numerical response and thus the growth rate of the predator population, as the ratio $B / P$ increases, the strength of the Allee effect increases; specifically when $B / P>\left(\theta N-r_{2}\right) / r_{2}$, then the growth rate of the predator population is negative (i.e. $\mathrm{d} P / \mathrm{d} t<0$ ).

The Lotka-Volterra system (1) has a trivial equilibrium which is a saddle point for all positive valued parameters, and a single non-trivial equilibrium at

$$
\left(N^{*}, P^{*}\right)=\left(\frac{r_{2}}{\theta}, \frac{r_{1}}{\delta}\right) \text {. }
$$

This non-trivial equilibrium represents coexistence of the predator and prey populations and is a centre for all positive parameter values. Thus, any initial condition $(N(0), P(0))$ such that $N(0), P(0)>0$ and $(N(0), P(0)) \neq\left(N^{*}, P^{*}\right)$ will result in solutions which trace along a closed orbit about $\left(N^{*}, P^{*}\right)$. See Figure $1($ a) for an example of such a cycle. 


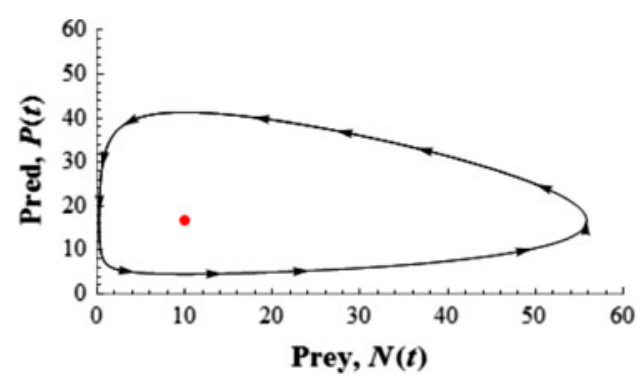

(a) Lotka-Volterra Model

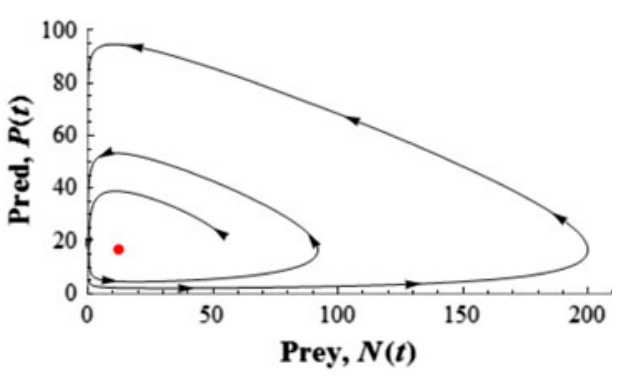

(b) Zhou et al. Model

Figure 1. Trajectories for the prey $(N)$ and predator $(P)$ populations described by (a) the Lotka-Volterra model given in System (1), and (b) the model proposed by Zhou et al. (2005) given in System (3). Parameter values: $r_{1}=.5, r_{2}=.1, \delta=.03, \theta=.01$ (for both trajectories), and $B=4$ for (b). In each graph, the location of the non-trivial equilibrium is indicated by the point.

Model (3) also has a trivial equilibrium which is a saddle point for all positive valued parameters, and a single non-trivial (coexistence) equilibrium at

$$
\left(N^{*}, P^{*}\right)=\left(\frac{r_{2}}{\theta} \frac{r_{1}+\delta B}{r_{1}}, \frac{r_{1}}{\delta}\right) \text {, }
$$

in which the prey population is larger than in the Lotka-Volterra system's non-trivial equilibrium. Furthermore, Zhou et al. (2005) showed that this equilibrium is an unstable focus for all positive-valued parameter sets which leads to boom and bust cycles with increasing amplitude in both populations. At some point, the height of the amplitude will become biologically untenable. See Figure 1(b) for an example trajectory.

It should be noted that the same model as System (3) was proposed by Bazykin (1998), though it was not explicitly described as adding an Allee effect. The model described by Zhou et al. (2005) in System (3) has been often cited as a method for introducing an Allee effect into the predator population of a predator-prey system (Courchamp et al., 2009; Flores \& González-Olivares, 2014; Lai, Liu, \& Lin, 2010; Terry, 2013, 2015; Verdy, 2010; Wang, Cai, \& Ma, 2013 for some examples) and as evidence that adding an Allee effect to the predator population of a predator-prey system can be destabilizing (Bompard, Amat, Fauvergue, \& Spataro, 2013; Boukal \& Berec, 2009; Boukal, Sabelis, \& Berec, 2007; Jang \& Diamond, 2007).

\section{Predator-prey model with an Allee effect \& intraspecific competition in the predator population}

We propose a modified version of the model presented by Zhou et al. (2005) that curtails the growth rate of the predator population at large densities relative to its prey source. Our model incorporates both an Allee effect and intraspecific competition into the numerical response of the predator population,

$$
\begin{aligned}
& \frac{\mathrm{d} N}{\mathrm{~d} t}=r_{1} N-\delta N P, \\
& \frac{\mathrm{d} P}{\mathrm{~d} t}=-r_{2} P+\theta N P\left(1-\frac{P}{\eta N}\right)\left(\frac{P}{P+B}\right),
\end{aligned}
$$


where $r_{1}, r_{2}, \delta$ and $\theta$ are positive-valued parameters as defined for the Lotka-Volterra model (1), and $B>0$ is the Allee effect constant as in Model (3). The parameter $\eta>0$ is a proportionality constant such that when the predator density $P$ is larger than $\eta N$, the numerical response term $f_{2}(N, P)=\theta N P\left(1-\frac{P}{\eta N}\right)\left(\frac{P}{P+B}\right)$ is negative. Thus, the ratio of the predator density to the prey density will determine when the predator population experiences decay due to competition between predators for prey, introduced in our model by the intraspecific competition factor $1-\frac{P}{\eta N}$. When the Allee effect constant $B$ is small, then the Allee effect will only cause reduced growth in the smallest predator densities. As the value of $B$ increases, the predator population experiences reduced growth for an increasing range of densities. However, when the predator densities are large relative to $B$, the Allee effect is negligible. In this case, when predator densities are also large relative to $\eta N$, then the intraspecific competition factor will dominate the numerical response. When predator densities are small relative to $\eta N$ while large relative to $B$, then the numerical response will behave like $\theta N P$, the numerical response from the Lotka-Volterra System (1). On the other hand, if predator population densities are small relative to $B$, then the Allee effect will slow the growth rate. In this case, when predator densities are also small relative to $\eta N$, then the intraspecific competition factor $1-\frac{P}{\eta N} \approx 1$ and, therefore has a negligible effect on the numerical response. When predator densities are large relative to $\eta N$ while small relative to $B$, then both factors decrease the numerical response and the effect is multiplicative allowing for impacts of the Allee effect and intraspecific competition to amplify each other. This can occur when the prey density is exceptionally low during periods of low predator density, in which case it is expected that the growth rate of the predator density would be very low and possibly negative provided the predator does not have an alternative prey source.

\subsection{Equilibria stability analysis}

Model (4) has four equilibrium points:

EQ 1: $N^{*}=0, P^{*}=0$.

$$
\begin{aligned}
& \text { EQ 2: } N^{*}=\frac{r_{2}}{\theta} \frac{r_{1}+\delta \dot{B}}{r_{1}}+\frac{r_{1}}{2 \delta \eta}, P^{*}=\frac{r_{1}}{\delta} . \\
& \text { EQ 3: } N^{*}=0, P^{*}=\frac{-r_{2} \eta-\sqrt{r_{2}^{2} \eta^{2}-4 B r_{2} \eta \theta}}{2 \theta} . \\
& \text { EQ 4: } N^{*}=0, P^{*}=\frac{-r_{2} \eta+\sqrt{r_{2}^{2} \eta^{2}-4 B r_{2} \eta \theta}}{2 \theta} .
\end{aligned}
$$

For EQ 3 and EQ 4 , let $\Delta=r_{2}^{2} \eta^{2}-4 B r_{2} \eta \theta$. When $\Delta<0$, then $P^{*} \notin \mathbb{R}$. When $\Delta \geq 0$, since all parameters are positive, then $P^{*}<0$. Thus, irregardless of the sign of $\Delta$, neither EQ 3 or EQ 4 are biologically tenable. Additionally, observe that the trivial equilibrium (EQ 1) of Model (4) is always a saddle point, since the eigenvalues of the Jacobian evaluated at EQ 1 have opposite signs, $\lambda_{1}=r_{1}$ and $\lambda_{2}=-r_{2}$. Therefore, we focus the remainder of the analysis on EQ 2, which we will refer to as the coexistence equilibrium.

Proposition 1 (Stability of EQ 2): Let $B_{c}(\eta)=r_{1}^{2} \theta /\left(r_{2} \delta^{2} \eta\right)$, where $r_{1}, r_{2}, \delta$, and $\eta$ are parameters as defined in System (4). The coexistence equilibrium (EQ 2) for System (4) 
is asymptotically stable when $B<B_{c}(\eta)$, a centre when $B=B_{c}(\eta)$, and unstable when $B>B_{c}(\eta)$.

Proof: The Routh-Hurwitz Conditions provide criteria for determining stability of an equilibrium and classifying the equilibrium type (Kot, 2003). Let $J$ represent the Jacobian evaluated at EQ 2. The trace and determinant of the Jacobian evaluated at EQ 2 are,

$$
\begin{aligned}
\operatorname{Tr}(J) & =\frac{B r_{2} \delta^{2} \eta-r_{1}^{2} \theta}{r_{1} \delta \eta+B \delta^{2} \eta}, \text { and } \\
\operatorname{det}(J) & =\frac{r_{1}^{2} r_{2} \delta \eta+B r_{1} r_{2} \delta^{2} \eta+r_{1}^{3} \theta}{r_{1} \delta \eta+B \delta^{2} \eta}
\end{aligned}
$$

The Routh-Hurwitz Conditions state that if $\operatorname{det}(J)<0$ then EQ 2 will be a saddle point (regardless of the sign of $\operatorname{Tr}(J)$ ), and that if $\operatorname{det}(J)>0$ then EQ 2 will be asymptotically stable when $\operatorname{Tr}(J)<0$, a centre when $\operatorname{Tr}(J)=0$, and unstable when $\operatorname{Tr}(J)>0$.

All parameters are positive, and thus $\operatorname{det}(J)>0$, i.e. there are no biologically-tenable parameters sets which cause EQ 2 to be a saddle point. Therefore we expect EQ 2 to be a stable node, an unstable node, a stable focus, an unstable focus or a centre.

If we solve $\operatorname{Tr}(J)=0$ for $B$ in terms of $\eta$, we obtain the curve

$$
B_{c}(\eta)=\frac{r_{1}^{2} \theta}{r_{2} \delta^{2} \eta}
$$

where the subscript $c$ denotes EQ 2 being a centre. Furthermore, EQ 2 will be asymptotically stable when $\operatorname{Tr}(J)<0$, which yields the condition $B<B_{c}(\eta)$, and EQ 2 will be unstable when $\operatorname{Tr}(J)>0$, which occurs when $B>B_{c}(\eta)$.

Note, $B_{c}(\eta)$ is the boundary between EQ 2 being asymptotically stable and unstable. The parameter regions for stability are depicted in Figure 2.

Proposition 2 (Classification of EQ 2): Let

$$
B_{n}(\eta)=-\frac{c}{b}, B_{n}^{+}(\eta)=\frac{-b+\sqrt{b^{2}-4 a c}}{2 a}, \text { and } B_{n}^{-}(\eta)=\frac{-b-\sqrt{b^{2}-4 a c}}{2 a}
$$

where the values $a=r_{2} \delta^{4} \eta^{2}\left(r_{2}-4 r_{1}\right), \quad b=-2 r_{1}^{2} \delta^{2} \eta\left(2 r_{1} \theta+r_{2}(4 \delta \eta+\theta)\right)$, and $c=r_{1}^{3}\left(r_{1} \theta^{2}-4 r_{1} \delta \eta \theta-4 r_{2} \delta^{2} \eta^{2}\right)$. Then the coexistence equilibrium (EQ 2) for System (4) can be classified as a node or focus by the following criteria:

(a) If $r_{2}=4 r_{1}$, then EQ 2 is a node when $B<B_{n}(\eta)$, and a focus otherwise.

(b) If $r_{2}<4 r_{1}$, then EQ 2 is a node when $B<B_{n}^{-}(\eta)$, and a focus otherwise.

(c) If $r_{2}>4 r_{1}$, then EQ 2 is a node when $B<B_{n}^{-}(\eta)$ or $B>B_{n}^{+}(\eta)$, and a focus otherwise.

Proof: The Routh-Hurwitz Conditions give that EQ 2 will be a node when $(\operatorname{Tr}(J))^{2}-$ $4 \operatorname{det}(J)>0$ and a focus when $(\operatorname{Tr}(J))^{2}-4 \operatorname{det}(J)<0$. Note that,

$$
(\operatorname{Tr}(J))^{2}-4 \operatorname{det}(J)=\frac{a B^{2}+b B+c}{\left(r_{1}+B \delta\right)^{2} \delta^{2} \eta^{2}}
$$




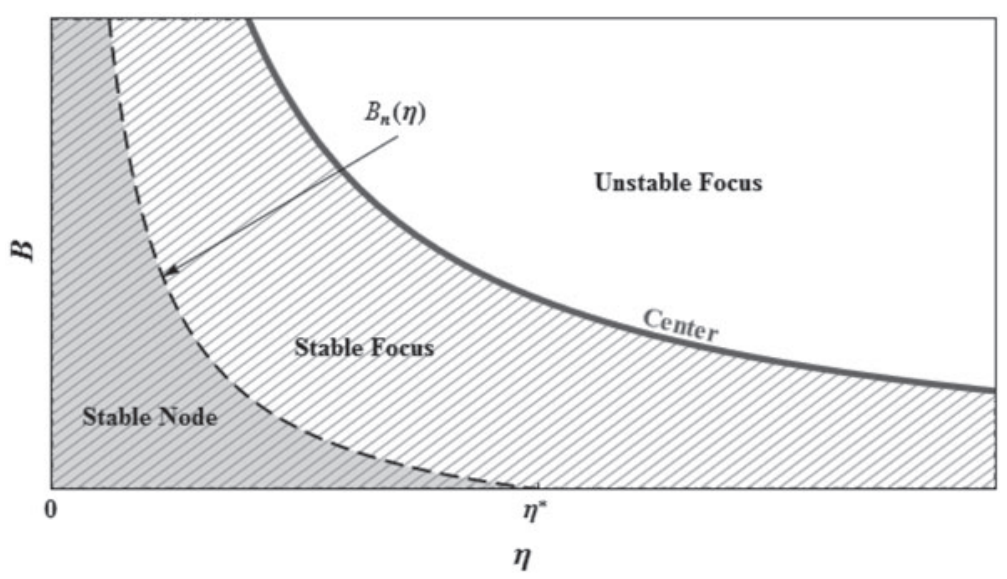

(a) $r_{2}=4 r_{1}$

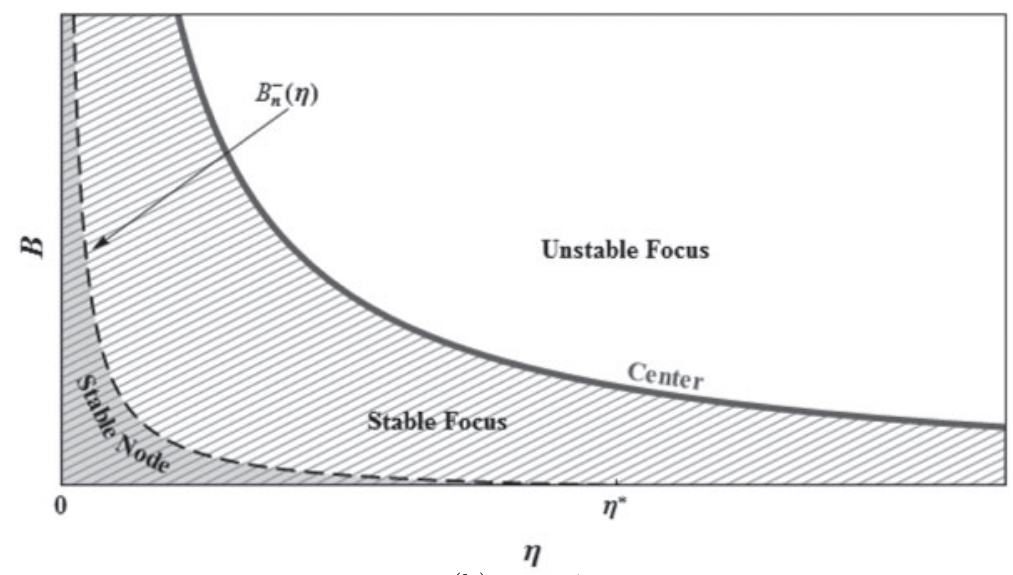

(b) $r_{2}<4 r_{1}$

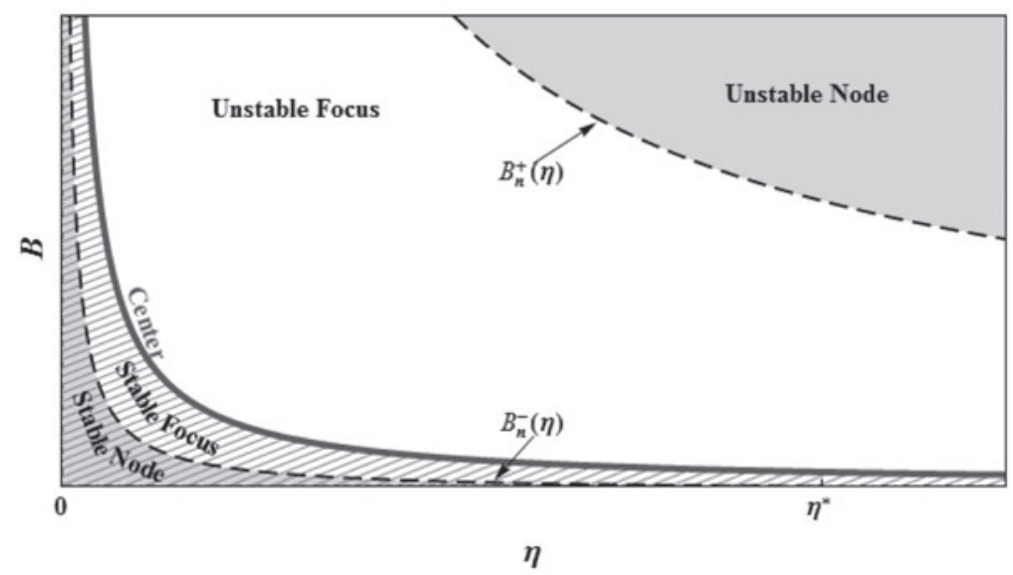

(c) $r_{2}>4 r_{1}$

Figure 2. The hatch-shaded region represents the parameter values for which the coexistence equilibrium of model (4) is stable. The solid-shaded region represents the parameter values for which the coexistence equilibrium is a node. The function $B_{n}(\eta)$ is given by Equation (10) and functions $B_{n}^{+}(\eta)$ and $B_{n}^{-}(\eta)$ are given by Equation (12). The solid grey curve is defined by $B_{c}(\eta)$ (Equation (7)), and $\eta^{*}$ is given by Equation (14). 
where

$$
\begin{gathered}
a=r_{2} \delta^{4} \eta^{2}\left(r_{2}-4 r_{1}\right), b=-2 r_{1}^{2} \delta^{2} \eta\left(2 r_{1} \theta+r_{2}(4 \delta \eta+\theta)\right), \\
\text { and } c=r_{1}^{3}\left(r_{1} \theta^{2}-4 r_{1} \delta \eta \theta-4 r_{2} \delta^{2} \eta^{2}\right) .
\end{gathered}
$$

The numerator of Equation (8) is quadratic in $B$, and the denominator is always positive. Thus, we can determine the sign of $(\operatorname{Tr}(J))^{2}-4 \operatorname{det}(J)$ by considering the sign of the quadratic $a B^{2}+b B+c$.

Let $f(B)=a B^{2}+b B+c$. First, note that since all parameters are positive, $a>0$ when $r_{2}>4 r_{1}$ and $b>0$ always. Since $c$ is quadratic in terms of $\eta$, we can determine that $c>0$ when

$$
0<\eta<\frac{\theta\left(\sqrt{r_{1}^{2}+r_{1} r_{2}}-r_{1}\right)}{2 r_{2} \delta} .
$$

Next, if $r_{2}=4 r_{1}$ (i.e. $a=0$ ), then $f(B)>0$ (i.e. EQ 2 is a node) when $B<-c / b$. Thus, in the case where $r_{2}=4 r_{1}$ the boundary between node and focus regions in the parameter space is given by

$$
B_{n}(\eta)=-\frac{c}{b}=\frac{r_{1}\left(\theta^{2}-4 \delta \eta \theta-16 \delta^{2} \eta^{2}\right)}{4 \delta^{2} \eta(8 \delta \eta+3 \theta)} .
$$

Note EQ 2 is a node when $B<B_{n}(\eta)$. Furthermore, note that when $r_{2}=4 r_{1}$,

$$
B_{c}(\eta)-B_{n}(\eta)=\frac{r_{1}\left(8 \delta^{2} \eta^{2}+6 \delta \eta \theta+\theta^{2}\right)}{2 \delta^{2} \eta(8 \delta \eta+3 \theta)}>0,
$$

since all parameters are assumed to be positive. Thus, when $r_{2}=4 r_{1}, B_{c}(\eta)>B_{n}(\eta)$, and consequently there exist no positive valued parameters sets such that EQ 2 is an unstable node (Figure 2(a)). Lastly, note that $B_{n}(\eta)>0$ when $0<\eta<\eta^{*}$ where

$$
\eta^{*}=\frac{\theta(\sqrt{5}-1)}{8 \delta} .
$$

Now, when $r_{2} \neq 4 r_{1}$ (i.e. $a \neq 0$ ) then the quadratic $f(B)$ has two roots

$$
B_{n}^{+}(\eta)=\frac{-b+\sqrt{b^{2}-4 a c}}{2 a} \text { and } B_{n}^{-}(\eta)=\frac{-b-\sqrt{b^{2}-4 a c}}{2 a} .
$$

Note that

$$
b^{2}-4 a c=16 r_{1}^{3} \delta^{4} \eta^{2}\left(r_{2}^{3} \delta^{2} \eta^{2}+3 r_{1} r_{2}^{2} \delta \eta \theta+r_{1}^{3} \theta^{2}+2 r_{1}^{2} r_{2} \theta^{2}\right)>0
$$

for all positive-valued parameters, and thus $B_{n}^{+}$and $B_{n}^{-}$are both real valued. The signs and relative sizes of $B_{n}^{+}(\eta)$ and $B_{n}^{-}(\eta)$ are determine by the signs of $a$ and $c$ and are given in Table 1. Additionally, if $r_{2}>4 r_{1}$ (i.e. $a>0$ ), then $f(B)>0$ when $B<B_{n}^{-}$or $B>B_{n}^{+}$; if $r_{2}<4 r_{1}$ (i.e. $a<0$ ), then $f(B)>0$ when $B_{n}^{+}<B<B_{n}^{-}$. However, these conditions can be reduced when we account for the sign of $c$ and require that $B>0$; Table 1 shows the simplifications in each case. Notice that if $r_{2}<4 r_{1}$ (i.e. $\left.a<0\right)$ and

$$
\eta>\frac{\theta\left(\sqrt{r_{1}^{2}+r_{1} r_{2}}-r_{1}\right)}{2 r_{2} \delta}
$$


Table 1. Signs and relative sizes of $B_{n}^{+}(\eta)$ and $B_{n}^{-}(\eta)$ as defined by Equation (12).

\begin{tabular}{ccccccc}
\hline & \multicolumn{3}{c}{ Sign of } & & & Conditions for \\
\hline$a$ & $c$ & $B_{n}^{+}(\eta)$ & $B_{n}^{-}(\eta)$ & & $B_{n}^{+}(\eta)>B_{n}^{-}(\eta)$ & $f(B)>0$ \\
\hline+ & + & + & + & & True & $0<B<B_{n}^{-}$or $B>B_{n}^{+}$ \\
+ & - & + & - & & True & $B>B_{n}^{+}$ \\
- & + & - & + & & False & $0<B<B_{n}^{-}$ \\
- & - & - & - & False & None \\
\hline
\end{tabular}

(i.e. $c<0$ ), there are no positive-valued parameter sets which cause $f(B)>0$; in other words, EQ 2 will be a focus.

It should be noted that when $r_{2} \neq 4 r_{1}$, the curve $B_{n}^{-}(\eta)$ has a root at

$$
\eta^{*}=\frac{\theta\left(\sqrt{r_{1}^{2}+r_{1} r_{2}}-r_{1}\right)}{2 r_{2} \delta},
$$

and the curve $B_{n}^{-}(\eta)>0$ when $0<\eta<\eta^{*}$. Thus, the condition for $B_{n}^{-}(\eta)>0$ is equivalent to the condition for $c>0$ (see Inequality (9)). Additionally, notice that $\eta^{*}>0$ for all positive-valued parameter sets, and when $r_{2}=4 r_{1}$ Equation (14) reduces to Equation (11).

Figure 2 shows the stability regions for the parameter conditions (a) $r_{2}=4 r_{1}$, (b) $r_{2}<4 r_{1}$, and (c) $r_{2}>4 r_{1}$. Note that the only case in which an unstable node occurs is when $r_{2}>4 r_{1}$ (Figure $2(\mathrm{c})$ ), and in each case stable nodes only occur when $0<\eta<\eta^{*}$, where $\eta^{*}$ is defined by Equation (14).

\subsection{Numerical results}

Figures 3-5 show sample trajectories of Model (4) with parameter sets where $r_{2}=4 r_{1}$ (Figure 3), $r_{2}<4 r_{1}$ (Figure 4), and $r_{2}>4 r_{1}$ (Figure 5). Parameter sets are chosen to illustrate the trajectory of the system given the coexistence equilibrium residing in one of the five potential stability regions shown in Figure 2: stable focus (Figures 3(a)5(a)), unstable focus (Figures 3(b)-5(b)), stable node (Figures 3(c)-5(c)), unstable node (Figure 5(d)), and centre (Figures 3(d)-5(e)). In each figure, all parameters are fixed with the exception of $B$ and $\eta$; all parameter values for each figure are given in Table 2 . This illustrates the impact the relative values of the strength of the Allee effect $B$ and the penalty for intraspecific competition $\eta$ have on the long term behaviour of the predator-prey system. Note, larger values for both $B$ and $\eta$ tend to result in the coexistence equilibrium being unstable.

Recall that the coexistence equilibrium can be an unstable node only in the case where $r_{2}>4 r_{1}$. Figure $5(\mathrm{~d})$ shows the trajectory of the predator and prey populations in the case where the coexistence equilibrium is an unstable node. This trajectory shows a slight rise in the predator density before it plummets to extinction, after which the prey population grows without bound. This eventual outcome of the predator population extinction while the prey population grows without bound also occurs in the cases where the coexistence equilibrium is an unstable focus (Figures 3(b), 4(b) and 5(b) for sample trajectories); 


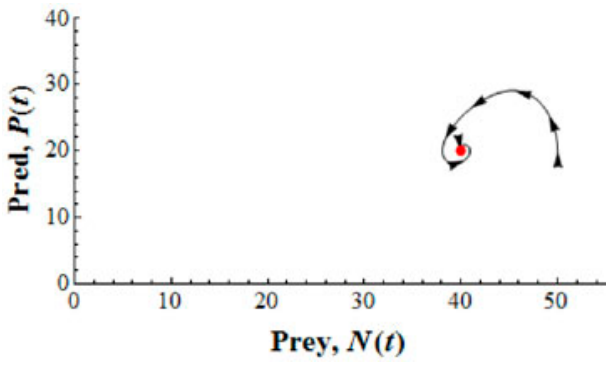

(a) Stable Focus; $B=30$

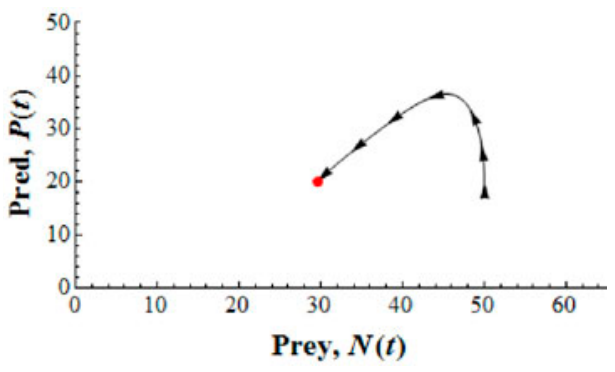

(c) Stable Node; $B=4$

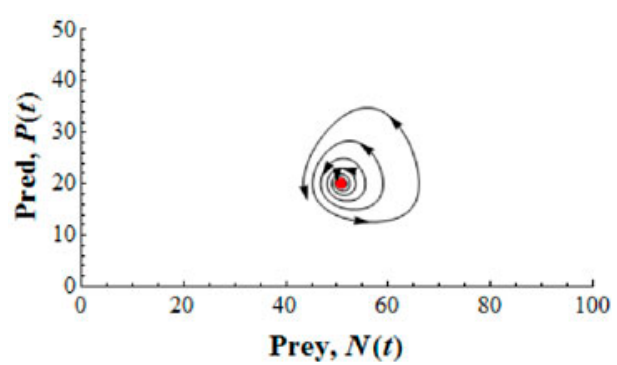

(b) Unstable Focus; $B=57$

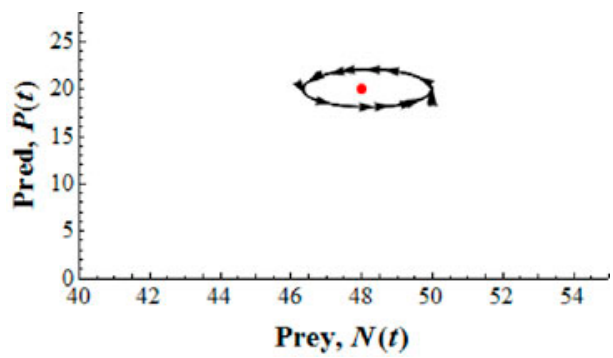

(d) Center; $B=50$

Figure 3. Trajectories of $(N(t), P(t))$ for model (4) with $r_{2}=4 r_{1}$. Each simulation uses $t \in[0,150]$, $N(0)=50, P(0)=20, r_{1}=.1, r_{2}=.4, \delta=.005, \theta=.05$, and $\eta=1$. The location of EQ 2 is indicated by the point.

Table 2. Parameter values used to generate Figures 3-5.

\begin{tabular}{|c|c|c|c|c|c|}
\hline & \multicolumn{2}{|c|}{ Stable } & \multirow[b]{2}{*}{ Center } & \multicolumn{2}{|c|}{ Unstable } \\
\hline & Node & Focus & & Node & Focus \\
\hline$r_{2}=4 r_{1}$ & Figure 3(c) & Figure $3(a)$ & Figure 3(d) & & Figure $3(b)$ \\
\hline$r_{1}=.1, r_{2}=.4$ & $\eta=1$ & $\eta=1$ & $\eta=1$ & & $\eta=1$ \\
\hline$\delta=.005, \theta=.05$ & $B=4$ & $B=30$ & $B=50$ & & $B=57$ \\
\hline$r_{2}<4 r_{1}$ & Figure 4(c) & Figure $4(a)$ & Figure 4(d) & & Figure 4(b) \\
\hline$r_{1}=.5, r_{2}=.3$ & $\eta=.1$ & $\eta=1$ & $\eta=1$ & & $\eta=3.8$ \\
\hline$\delta=.03, \theta=.02$ & $B=5$ & $B=1$ & $B=18.5185$ & & $B=5$ \\
\hline$r_{2}>4 r_{1}$ & Figure 5(c) & Figure 5(a) & Figure 5(e) & Figure 5(d) & Figure 5(b) \\
\hline$r_{1}=.03, r_{2}=.3$ & $\eta=.75$ & $\eta=1$ & $\eta=1$ & $\eta=2$ & $\eta=1$ \\
\hline$\delta=.002, \theta=.02$ & $B=3$ & $B=14$ & $B=15$ & $B=5$ & $B=16$ \\
\hline
\end{tabular}

however, the trajectories are not simulated over a long enough period of time to show this eventual outcome.

Lastly, it should be noted that when the coexistence equilibrium is a centre, both populations display periodic cycling as shown in Figures 3(d), 3(d), and 5(e). However, even slight perturbations of the parameter values will cause the equilibrium to shift to a focus whose stability depends on the direction of the perturbations (Figure 2). For example, Figure 5(e) shows a centre when $r_{2}>4 r_{1}, \eta=1$ and $B=15$. Notice, when the value of $B$ is increased to $B=16$ (without changing the value of $\eta$ or any other parameters), the regular oscillations of the centre become oscillations with increasing amplitude, i.e. an unstable focus (Figure 5(b)). Additionally, notice that when the value of $B$ is decreased to $B=14$ 


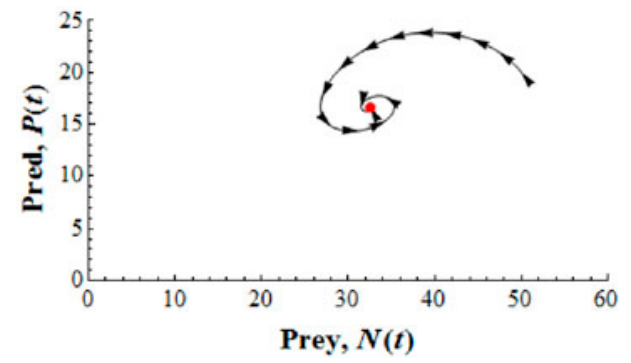

(a) Stable Focus; $\eta=1, B=1$

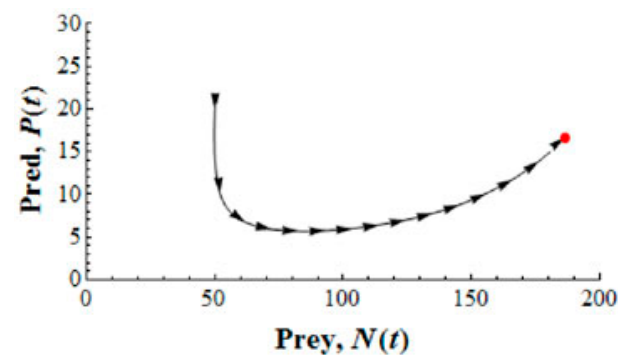

(c) Stable Node; $\eta=0.1, B=5$

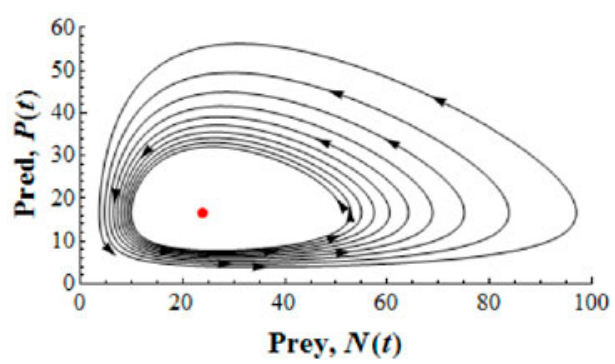

(b) Unstable Focus; $\eta=3.8, B=5$

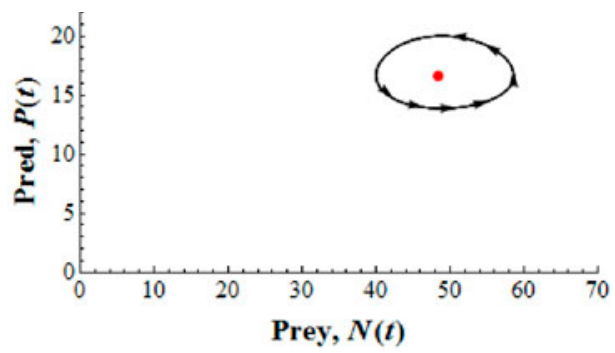

(d) Center; $\eta=1, B=18.5185$

Figure 4. Trajectories of $(N(t), P(t))$ for model (4) with $r_{2}<4 r_{1}$. Each simulation uses $t \in[0,150]$, $N(0)=50, P(0)=20, r_{1}=.5, r_{2}=.3, \delta=.03$, and $\theta=.02$. The location of EQ 2 is indicated by the point.

(without changing any other parameters), the coexistence equilibrium becomes a stable focus, and over time the oscillating populations will approach a steady state (Figure 5(a)). It should be noted, any increase in $B$, however small, would have resulted in the coexistence equilibrium becoming an unstable focus, and any decrease in $B$ would have resulted in the equilibrium becoming a stable focus. Thus, in an environment in which the values of $\eta$ and $B$ may be prone to perturbation, one should not expect the coexistence equilibrium to remain a centre over a long-time horizon.

\section{Discussion}

Our proposed model modifies the numerical response of the predators to their prey from the Lotka-Volterra model (1) by decreasing the numerical response for relatively small predator densities (Allee effect) and causing the numerical response to become negative for sufficiently large ratios of predator densities to prey densities (intraspecific competition). These modifications to the numerical response led our model to have quantitatively different coexistence equilibrium points and qualitatively different coexistence equilibrium stability outcomes than the Zhou et al. and Lotka-Volterra models. Table 3 shows a comparison of the numerical responses of the predator population to its prey and the resulting coexistence equilibrium of each model. 


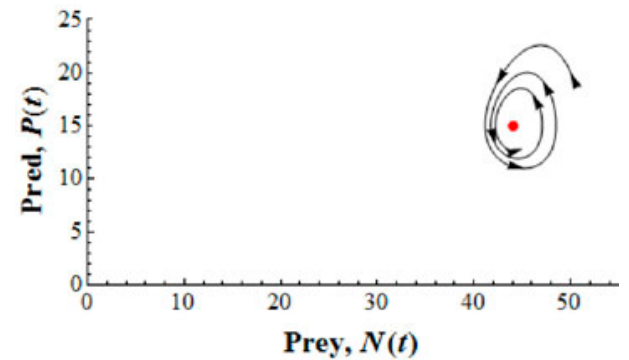

(a) Stable Focus; $\eta=1, B=14$

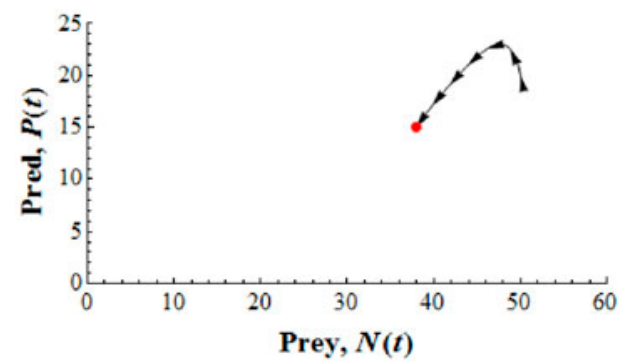

(c) Stable Node; $\eta=0.75, B=3$

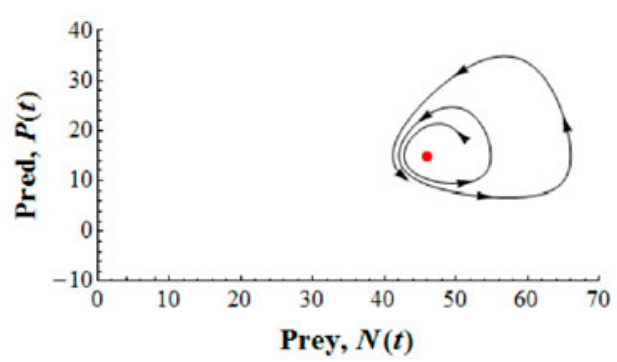

(b) Unstable Focus; $\eta=1, B=16$

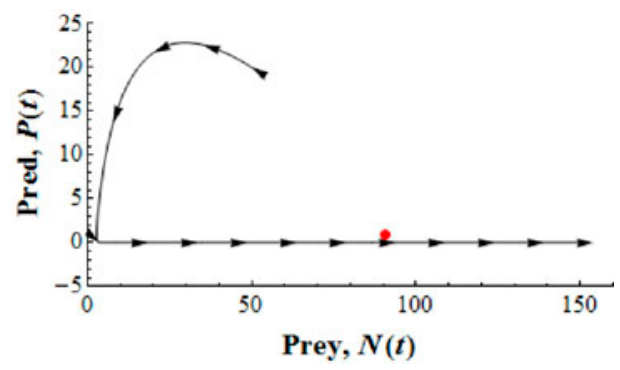

(d) Unstable Node; $\eta=2, B=5$

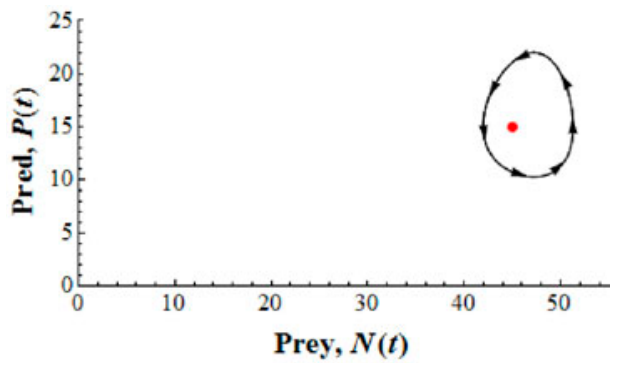

(e) Center; $\eta=1, B=15$

Figure 5. Trajectories of $(N(t), P(t))$ for model (4) with $r_{2}>4 r_{1}$. Each simulation uses $t \in[0,150]$, $N(0)=50, P(0)=20, r_{1}=.03, r_{2}=.3, \delta=.002$, and $\theta=.02$. The location of EQ 2 is indicated by the point.

Table 3. The numerical response terms of the predator population to its prey, $f_{2}(N, P)$ and the coexistence equilibria for each of the models discussed: the Lotka-Volterra model defined by system (1); the Zhou et al. model defined by system (3); and the novel model proposed here defined by system (4).

\begin{tabular}{lcr}
\hline Model & $f_{2}(N, P)$ & Coexistence EQ $\left(N^{*}, P^{*}\right)$ \\
\hline Lotka-Volterra (1) & $\theta N P$ & $\left(\frac{r_{2}}{\theta}, \frac{r_{1}}{\delta}\right)$ \\
Zhou et al. (3) & $\theta N P\left(\frac{P}{P+B}\right)$ & $\left(\frac{r_{2}}{\theta} \frac{r_{1}+\delta B}{r_{1}}, \frac{r_{1}}{\delta}\right)$ \\
Our model (4) & $\theta N P\left(1-\frac{P}{\eta N}\right)\left(\frac{P}{P+B}\right)$ & $\left(\frac{r_{2}}{\theta} \frac{r_{1}+\delta B}{r_{1}}+\frac{r_{1}^{2}}{2 \delta \eta}, \frac{r_{1}}{\delta}\right)$ \\
\hline
\end{tabular}




\subsection{Size of the prey population at the coexistence equilibrium}

Note that the prey population at the coexistence equilibrium for the Zhou et al. model (3) is proportionally larger than that of the Lotka-Voleterra model (1). Furthermore, the prey population at the coexistence equilibrium for our model (4) is even larger than that of the Zhou et al. model. Thus,

$$
\frac{r_{2}}{\theta}<\frac{r_{2}}{\theta} \frac{r_{1}+\delta B}{r_{1}}<\frac{r_{2}}{\theta} \frac{r_{1}+\delta B}{r_{1}}+\frac{r_{1}^{2}}{2 \delta \eta} .
$$

Since our model restricts the predator population from becoming excessively large through the mechanism of intraspecific competition, at the coexistence equilibrium the prey population is able to attain a large density despite the fact that the predator density is the same at the coexistence equilibrium for all three models.

\subsection{Coexistence equilibrium stability outcomes}

In comparison to the Zhou et al. model (3) that, for all biologically reasonable parameter values, has an unstable focus and the Lotka-Volterra model (1) that, for all biologically reasonable parameter values, has a centre, the stability of our model (4) is parameter dependent. In particular, the relationship between $B$ (the Allee effect constant) and $\eta$ (the intraspecific competition proportionality constant) determines the stability of the coexistence equilibrium. Note, the larger the value of $B$, the greater the density of predators required to maintain a positive predator per capita growth rate. The larger the value of $\eta$, the smaller the density of prey may be before the predator population experiences negative per capita growth rates as a result of intraspecific competition. If $B$ is large, then $\eta$ must be relatively small (and vice versa) to maintain stability (see hatch-shaded regions in Figure 2). Additionally, if both $B$ and $\eta$ are relatively small, then stability is maintained.

\subsection{Penalty on large predator population densities}

In the Zhou et al. model (3), the addition of an Allee effect term on the numerical response caused the oscillations of the predator and prey densities to have increasing amplitudes, resulting in an unstable focus. Biologically, it is expected that very dense populations will be disadvantageous, and so it is reasonable to incorporate a penalty on the numerical response for large predator population densities relative to the density of their food source. A penalty for intraspecific competition used in our model (4), can in some cases, dampen the potentially unbounded growth caused by the Allee effect term; this penalty creates biologically tenable parameter sets that result in a stable coexistence equilibrium.

\subsection{Model limitations \& extensions}

Our model (4) produces an unstable node for some biologically reasonable parameter sets. In these cases, the predator population goes extinct after which the prey population increases without bound (Figure 5(d)). Eventually, the density of the prey population will become untenable. Instead, the prey population would approach a carrying capacity dependent on the limitations on the space and resources of the habitat. The unbounded 
prey growth occurs because, although our model (4) includes a penalty for large predator populations, we did not impose a similar penalty on the prey population. A future extension of this model could include this feature.

The penalty used for large predator population densities addresses decreased per capita growth due to intraspecific competition, which depends on the ratio of predator to prey population densities. However, other penalties may exist for large population sizes that do not depend on the population density of another species. For instance, there may be a spatial limitation for the predator population, or territorial species may spend less time hunting due to intraspecific distraction (Kratina, Vos, Bateman, \& Anholt, 2009). A future model could explore another type of penalty for large populations or combination of penalties.

The Lotka-Volterra model (1) describes simplified predator-prey dynamics well, yet we know many additional forces may be present which can alter the long-term behaviour of the system. Ecologists and conservationists have produced empirical evidence supporting the presence of the Allee effect (Courchamp, Clutton-Brock, \& Grenfell, 1999; Kunin, 1992) and intraspecific competition (Knowlton, 1992; Kratina et al., 2009; Morin, 1986), with specific examples of varied increases in intraspecific aggression when the food source is scarce (Clark et al., 1999; Fox, 1975; Polis, 1981). Additionally, many theoretic models have been constructed to examine the effects of each of these forces on ecological systems (Bodine, Gross, \& Lenhart, 2008; De Silva \& Jang, 2015; Jang, 2010, 2013; McCarthy, 1997; Ramos-Jiliberto, 2003; Wang, Liang, \& Wang, 1999; Wittmann, Hutzenthaler, Gabriel, \& Metzler, 2013 for some examples). We have presented a new theoretic model that includes these two empirically driven forces, which results in the parameter-dependent stability of the coexistence equilibrium. Future studies of predator-prey systems can be used to identify the particular parameter values that best model the density-dependent dynamics of specific systems, and the theory presented here can be used to predict the long-term stability of that system.

\section{Acknowledgements}

EB thanks Elysia Hassen and Rebecca Olivarez for their work on predator-prey models which eventually inspired this project. Hassen and Olivarez explored a variety of unsuitable predator-prey models which paved the way for the model presented here.

\section{Disclosure statement}

No potential conflict of interest was reported by the authors.

\section{References}

Allee, W. (1941). Integration of problems concerning protozoan populations with those of general biology. The American Naturalist, 75, 473-487.

Aulisa, E., \& Jang, S. (2014). Continuous-time predator-prey systems with Allee effects in the prey. Mathematics and Computers in Simulation, 105, 1-16.

Bazykin, A. (1998). Nonlinear dynamics of interacting populations. New Jersey: World Scientific.

Bodine, E. N., Gross, L. J., \& Lenhart, S. (2008). Optimal control applied to a model for species augmentation. Mathematical Biosciences and Engineering, 5, 669-680. 
Bompard, A., Amat, I., Fauvergue, X., \& Spataro, T. (2013). Host-parasitoid dynamics and the success of biological control when parasitoids are prone to Allee effects. PLOS One, 8, e76768.

Boukal, D., \& Berec, L. (2009). Modelling mate-finding Allee effects and populations dynamics, with applications in pest control. Population Ecology, 51, 445-458.

Boukal, D., Sabelis, M., \& Berec, L. (2007). How predator functional responses and Allee effects in prey affect the paradox of enrichment and population collapses. Theoretical Population Biology, $72,136-147$.

Clark, M. E., Wolcott, T. G., Wolcott, D. L., \& Hines, A. H. (1999). Intraspecific interference among foraging blue crabs Callinectes sapidus: Interactive effects of pradator density and prey patch distribution. Marine Ecology Progress Series, 178, 69-78.

Courchamp, F., Berec, L., \& Gascoigne, J. (2009). Allee effects in ecology and conservation. New York, NY: Oxford Biology, Oxford University Press.

Courchamp, F., Clutton-Brock, T., \& Grenfell, B. (1999). Inverse density dependence and the Allee effect. Tree, 14, 405-410.

De Silva, M., \& Jang, S. (2015). Competitive exclusion and coexistence in a Lotka-Volterra competition model with Allee effects and stocking. Letters in Biomathematics, 2, 56-66.

Flores, J., \& González-Olivares, E. (2014). Dynamics of a predator-prey model with Allee effect on prey and ratio-dependent functional response. Ecological Complexity, 18, 59-66.

Fox, L. R. (1975). Capredation in natural populations. Annual Review of Ecology and Systematics, 6, 87-106.

Gascoigne, J. C., \& Lipcius, R. N. (2004). Allee effects driven by predation. Journal of Applied Ecology, $41,801-810$.

Gusset, M., \& Macdonald, D. W. (2010). Group size effects in cooperatively breeding African wild dogs. Animal Behaviour, 79, 425-428.

Jang, S. (2010). Dynamics of an age-structured population with Allee effects and harvesting. Journal of Biological Dynamics, 4, 409-427.

Jang, S. (2013). On the Lotka-Volterra competition system with Allee effects. Computational \& Applied Mathematics, 32, 179-189.

Jang, S., \& Diamond, S. (2007). A hostparasitoid interaction with Allee effects on the host. Computational \& Applied Mathematics, 53, 89-103.

Knowlton, N. (1992). Threshold and multiple stable states in coral reef community dynamics. American Zoologist, 32, 674-682.

Kot, M. (2003). Elements of mathematical ecology. New York, NY: Cambridge University Press.

Kratina, P., Vos, M., Bateman, A., \& Anholt, B. R. (2009). Functional repsonses modified by predator density. Oecologia, 159, 425-433.

Kunin, W. E. (1992). Defect and reproductive success in wild populations of Diplotaxis erucoides (Brassicaceae). Oecologia, 91, 129-133.

Lai, X., Liu, S., \& Lin, R. (2010). Rich dynamical behaviours for predator-prey model with weak Allee effect. Applicable Analysis, 89, 1271-1292.

Lotka, A. J. (1925). Elements of physical biology. Baltimore: Williams \& Wilkins.

McCarthy, M. A. (1997). The Allee effect, finding mats and theoretical models. Ecological Modelling, 103, 99-102.

Morin, P. J. (1986). Interactions between intraspecific competition and predation in an amphibian predator-prey system. Ecology, 67, 713-720.

Polis, G. A. (1981). The evolution and dynamics of intraspecific predation. Annual Review of Ecology and Systematics, 12, 225-251.

Purves, W. K., Sadava, D., Orians, G. H., \& Heller, H. C. (2001). Life: The science of biology (6th ed.). Sunderland, MA: Sinauer Associates.

Ramos-Jiliberto, R. (2003). Population dynamics of prey exhibiting inducible defenses: The role of associated costs and density-dependence. Theoretical Population Biology, 64, 221-231.

Terry, A. J. (2013). Prey resurgence from mortality events in predator-prey models. Nonlinear Analysis: Real, 14, 2180-2203.

Terry, A. J. (2015). Predator-prey models with component Allee effect for predator reproduction. The Journal of Mathematical Biology, 71, 1325-1352. 
Verdy, A. (2010). Modulation of predator-prey interactions by the Allee effect. Ecological Modelling, $221,1098-1107$.

Volterra, V. (1926). Variations and fluctuations of the number of individuals in animal species living together. Journal Consultation Per International Ent Mer, 3, 3-51.

Wang, G., Liang, X.-G., \& Wang, F.-Z. (1999). The competative dynamics of popuations subject to an Allee effect. Ecological Modelling, 124, 183-192.

Wang, X., Cai, Y., \& Ma, H. (2013). Dynamics of a diffusive predator-prey model with Allee effect on predator. Discrete Dynamics in Nature and Society, 2013, 1-10.

Wittmann, M. J., Hutzenthaler, M., Gabriel, W., \& Metzler, D. (2013). Ecological and genetic effects of introduced species on their native competitors. Theoretical Population Biology, 84, 25-35.

Zhou, S., Liu, Y., \& Wang, G. (2005). The stability of predator-prey systems subject to the Allee effects. Theoretical Population Biology, 67, 23-31. 